\title{
The European Network of Laboratories: Visual Double Stars.
}

\author{
E. OBLAK ${ }^{1}$, A.N. ARGUE ${ }^{2}$, P. BROSCHE ${ }^{3}$, J. CUYPERS 4 , \\ J. DOMMANGET ${ }^{4}$, A. DUQUENNOY ${ }^{5}$, M. FROESCHLE ${ }^{6}$, \\ M. GRENON ${ }^{5}$, J.-L. HALBWACHS ${ }^{7}$, G. JASNIEWICZ ${ }^{7}$, \\ P. LAMPENS ${ }^{4}$, J.C. MERMILLIOD ${ }^{8}$, F. MIGNARD ${ }^{6}$, \\ D. SINACHOPOULOS ${ }^{4}$, W. SEGGEWISS ${ }^{3}, \&$ E. VAN DESSEL ${ }^{4}$ \\ 1 Observatoire de Besançon, France \\ 2 Institute of Astronomy, Cambridge, United Kingdom \\ 3 Observatorium Hoher List, Bonn, Germany \\ 4 Royal Observatory of Belgium, Brussels, Belgium \\ ${ }^{5}$ Observatoire de Genève, Sauverny, Switzerland \\ 6 Observatoire de la Côte d'Azur, Grasse, France \\ 7 Observatoire de Strasbourg, France \\ ${ }^{8}$ Institut Astronomique de Lausanne, Sauverny, Switzerland
}

\section{INTRODUCTION.}

The study of double stars has since long been recognized as a basic key to the understanding of star formation and stellar evolution. Moreover, close visual double stars have always been systematically neglected in photometric observational programmes although they contain an important part of physically associated systems. It is then timely to organize major observational programmes of these objects for a number of good reasons:

1. The frequency of double stars is continuously reviewed and the rate of their detection is steadily increasing - both from ground-based and space observations - in such a way that they no longer can be discarded in any models of galactic structure;

2. Space observations (HIPPARCOS, HST) significantly improve the quality and the importance of stellar samples and permit to better take into account some of the selection effects;

3. The high-quality astrometric (and also photometric) data that will be provided for such systems by the space observations should be matched with accurate and homogeneous complementary astrophysical information such as colour indices and spectral classification. Such information for close visual double stars is unfortunately almost non-existent but are now being more easily accessible with the use of CCD detectors.

\section{THE EUROPEAN NETWORK OF LABORATORIES.}

In order to remedy the lack of photometric data on visual double stars, a European network of laboratories "Visual Double Stars" was created in August 1990 with the approval and funding of the French "Ministère de la Recherche et de la Technologie" for a period of three years (the duration of activities could be extendable to five years). The structure of the network is described in Table 1. 
TABLE 1. European Network of Laboratories: "Visual Double Stars"

\begin{tabular}{|l|l|}
\hline BELGIUM & $\begin{array}{l}\text { Royal Observatory of Belgium } \\
\text { Obserwartorium Hoher List der Universitats- } \\
\text { Sternwarte Bonn } \\
\text { "Observatorio Calar Alto" } \\
\text { Observatoire de Besançon }\end{array}$ \\
Observatoire de la Côte d'Asur \\
ObwTZERLAND & $\begin{array}{l}\text { Observatoire de Strasbourg } \\
\text { Observatoire de Genève } \\
\text { Institut Astronomique de l'Université de Lausanne } \\
\text { Institute of Astronomy, University of Cambridge and } \\
\text { "Observatory Roque de Los Muchachos", La Palms }\end{array}$ \\
\hline
\end{tabular}

The definition of the scientific goals and the preparation of the observational programme were established for both hemispheres during several round table discussions. These major observational campaigns become feasible thanks to specific contributions of the different member countries of the network: we dispose of observing opportunities at the observatories of Calar Alto (Germany), La Palma (United Kingdom), "Observatoire de Haute-Provence" (France), Jungfraujoch (Switzerland), La Silla (ESO) and easy access to the data bases of "Centre de Données Astronomiques de Strasbourg" and of Lausanne.

\section{OBSERVATIONAL PROGRAMME.}

Less than $10 \%$ of the components of systems in the astrometric "Catalogue des Composantes Doubles et Multiples" (CCDM; Dommanget 1989) have accurate photometric magnitudes. A photometric catalogue of components of multiple systems containing measurements in mainly the UBV, Geneva, and Stroemgren photometric systems has been used to define the programme in combination with double star catalogues (CPSDM; Oblak, 1988).

At ESO, a key-programme for photometry has been established for a period of three years, allowing us to start immediately observations in the southern hemisphere.

Highest priority is given to the systems observed by the satellite HIPPAR$\operatorname{COS}$ (about 14,000) since we will get accurate values of their proper motions and parallaxes. For the same reasons, we intend to include the new visual binaries discovered by HIPPARCOS as well.

Details on the observational programme can be found in the accompanying paper "Observational programme for classical and CCD photometry of visual double and multiple systems" by Oblak \& Lampens.

\section{SCIENTIFIC AIMS.}

Observations by conventional aperture and CCD photometries in both hemispheres should allow us to investigate a more complete and unbiased sample of visual double stars in a given spatial volume. 
By focusing on the HIPPARCOS pairs in the Input Catalogue, the definition of such a spatial sample is made more easily feasible. On the other hand, we will be able to provide the colour indices of the components needed to solve certain problems in the reduction of the satellite data. In addition, the scientific returns also concern:

1. the recognition of pairs that may be physically associated;

2. a better knowledge of the statistics and the principal characteristics of visual binaries: their frequency (e.g., Halbwachs, 1986), spatial distribution, real separations, magnitude differences, orbits and mass ratios as a function of spectral type and/or age, their distribution in the HR diagram. Knowledge of the real frequency is crucial in the development of synthetic models of stellar populations (Robin \& Crézé 1986, Gilmore et al. 1989).

3. binary formation and evolution; the mass-luminosity relation, the evolution of orbital parameters, the relation between the kinematic and the physical characteristics of systems in the Galaxy (Oblak 1983).

4. calibration problems (also for the wide pairs): calibration of the absolute magnitudes, stellar abundances, interstellar extinction through exploitation of the common ages, distances and chemical compositions of the components.

\section{FORTHCOMING EXTENSIONS.}

In order to have a more complete and less biased sample, we intend to introduce the systems not yet repertoriated in the HIPPARCOS Input Catalogue and listed in recent double star catalogues (Couteau 1992, Worley \& Douglas 1984 or its new version, Worley 1989). We also hope to include the new double stars with separations greater than one arcsecond detected in the HIPPARCOS mission.

For a more complete evaluation of multiplicity in our survey, the information on spectroscopic and speckle interferometric duplicity will be introduced as well (McAlister \& Hartkopf, 1984, 1988).

The obtained photometric data will be integrated into the data base of the "Centre de Données Astronomiques de Strasbourg".

\section{REFERENCES}

Couteau, P. 1992, Catalogue to be published in "Centre de Données Astronomiques de Strasbourg"

Dommanget, J., 1989, Star Catalogue: A Centennial Tribute to A. N. Vyssotsky, ed. A.G.D. Philip and A.R. Upgren, 77

Gilmore, G., Wyse, R., \& Kuijen, K., 1989, ARA $\& A, 25,555$

Halbwach8, J.L., 1986, $A \& A S, 66,131$

McAlister, H.A. \& Hartkopf, W.I. 1984, Catalog of Interferometric Measurements of Binary Stars, CHARA Contribution No 1

McAlister, H.A. \& Hartkopf, W.I. 1988, Second Catalog of Interferometric Measurements of Binary Stars, CHARA Contribution No 2

Oblak, E. $1983, A \& A, 123,238$

Oblak, E. 1988, Ap\&SS, 142, 31

Robin, A. \& Crézé, M. 1986, $A \& A, 157,71$

Worley, C. E. \& Douglas, G. G. 1984, The Washington Double Star Catalog

Worley, C. E. 1989, Publ. U.S.N.O., Vol. 25 